\title{
Pattern formation in a reaction-diffusion ratio-dependent predator-prey model
}

\author{
Marcos Lizana and Julio J. Marín V.
}




\title{
PATTERN FORMATION IN A REACTION-DIFFUSION RATIO-DEPENDENT PREDATOR-PREY MODEL
}

\author{
MARCOS LIZANA AND JULIO J. MARÍN V.
}

[Received: December 7, 2004]

\begin{abstract}
Aвstract. In this paper we study the effect of diffusion on the stability of the equilibria in a reaction-diffusion ratio-dependent predator-prey model and we explore under which parameter values Turing instability can occur giving rise to nonuniform stationary solutions. Moreover, their stability is studied.
\end{abstract}

Mathematics Subject Classification: 35K57, 92D25

Keywords: Turing instability, pattern formation, ratio-dependent predator-prey, reactiondiffusion system

\section{InTRODUCTION}

In this paper we are going to study the following reaction diffusion ratio dependent predator prey model

$$
\begin{aligned}
& \frac{\partial N}{\partial t}=D_{1} \Delta N+a N\left(1-\frac{N}{K}\right)-\frac{c N P}{m P+N}, \quad x \in \Omega, t>0, \\
& \frac{\partial P}{\partial t}=D_{2} \Delta P+P\left(-d+\frac{f N}{m P+N}\right), \quad x \in \Omega, t>0,
\end{aligned}
$$

subject to the Neumann boundary conditions

$$
\frac{\partial N}{\partial \eta}=\frac{\partial P}{\partial \eta}=0, \quad x \in \partial \Omega, \quad t>0,
$$

and initial conditions

$$
N(x, 0)=\varphi_{1}(x) \geq 0, \quad P(x, 0)=\varphi_{2}(x) \geq 0, \quad x \in \Omega .
$$

where $a, K, c, m, f, d$ are positive constants and $N(x, t), P(x, t)$ represent the population density of prey and predator at $x \in \Omega$ and at time $t$ respectively. The prey grows with intrinsic growth rate $a$ and carrying capacity $K$ in the absence of predation. The predator consumes the prey with functional response of Michaelis-Menten type $c u y /(m+u), u=x / y$ and contributes to its growth with rate $f u y /(m+u)$. The 
constant $d$ is the death rate of predator, and $D_{i}>0$ are constants, $i=1,2$; while $\Delta$ denotes the Laplace operator in $\Omega \subset \mathbb{R}^{n}, \Omega$ bounded and connected.

The motivation to consider the above described model comes from growing evidence $[1,2,4,7]$ that in some situations, specially when predators have to search for food and therefore have to share or compete for food, a more suitable general predator prey theory should be based on the so-called ratio-dependent theory, which can be roughly stated as that the per capita predator growth rate should be a function of the ratio of prey to predator abundance. This is supported by numerous field and laboratory experiments and observations [2-4].

Hsu et al. in [11] perform a global analysis of the Michaelis-Menten-type ratiodependent predator-prey system without diffusion. Moreover, they discuss the main differences between the classical predator-prey models and the ratio dependent predatorprey system. In particular they brought into discussion the well-known "paradox of enrichment" or equivalently "the biological control paradox."

In this paper we will study the effect of diffusion on the stability of the equilibria in a reaction-diffusion ratio-dependent predator-prey model and we explore under which parameter values Turing instability can occur giving rise to non-uniform stationary solutions. Their stability is studied. Moreover, we give a comprehensive description, under which parameter values this pattern formation arises. In the concluding remark we will discuss the differences between the dynamics of this model and the classical one.

\section{Preliminaries}

For simplicity, we undimensionalize the system (1.1) with the scaling $t \rightarrow a t$, $N \rightarrow N / K, P \rightarrow m P / K$. Then system (1.1) takes the form

$$
\begin{array}{ll}
\frac{\partial N}{\partial t}=d_{1} \Delta N+N(1-N)-\frac{s N P}{P+N}, & x \in \Omega, t>0, \\
\frac{\partial P}{\partial t}=d_{2} \Delta P+\delta P\left(-r+\frac{N}{P+N}\right), & x \in \Omega, t>0,
\end{array}
$$

where

$$
s=\frac{c}{m a}, \quad \delta=\frac{f}{a}, \quad r=\frac{d}{f}, \quad d_{1}=\frac{D_{1}}{a}, \quad d_{2}=\frac{D_{2}}{a} .
$$

We will show that the reaction-diffusion system (2.1) generates a dynamical system and it is biologically well-posed on a suitable Banach space.

Let us set $F=\left(F_{1}, F_{2}\right), U=(N, P)$ and $D=\operatorname{diag}\left[d_{1}, d_{2}\right]$, where

$$
F_{1}(N, P)=N(1-N)-\frac{s N P}{N+P}, \quad F_{2}(N, P)=\delta P\left(-r+\frac{N}{P+N}\right) .
$$


Henceforth, considering also an initial condition, system (2.1) can be rewritten as

$$
\begin{aligned}
\frac{\partial U(x, t)}{\partial t} & =D \Delta U(x, t)+F(U), \quad x \in \Omega, \quad t>0 \\
\frac{\partial U}{\partial \eta}(x, t) & =0, \quad x \in \partial \Omega, \quad t>0 \\
U(x, 0) & =\varphi(x), \quad x \in \Omega .
\end{aligned}
$$

Let $X$ be the Banach space $X_{1} \times X_{2}$, where $X_{i}=C(\bar{\Omega}), i=1,2$. The norm on $X$ is defined by $|\varphi|=\left|\varphi_{1}\right|+\left|\varphi_{2}\right|$. Let $A_{N}^{0}$ and $A_{P}^{0}$ be the differential operators $A_{N}^{0} N=d_{1} \Delta N$ and $A_{P}^{0} P=d_{2} \Delta P$, defined on the domains $D\left(A_{N}^{0}\right)$ and $D\left(A_{P}^{0}\right)$, respectively:

$$
\begin{aligned}
& D\left(A_{N}^{0}\right)=\left\{N \in C^{2}(\Omega) \cap C^{1}(\bar{\Omega}): A_{N}^{0} N \in C(\bar{\Omega}), \frac{\partial N}{\partial \eta}(x)=0, x \in \partial \Omega\right\}, \\
& D\left(A_{P}^{0}\right)=\left\{P \in C^{2}(\Omega) \cap C^{1}(\bar{\Omega}): A_{P}^{0} P \in C(\bar{\Omega}), \frac{\partial P}{\partial \eta}(x)=0, x \in \partial \Omega\right\} .
\end{aligned}
$$

The closures $A_{N}$ of $A_{N}^{0}$, and $A_{P}$ of $A_{P}^{0}$ in $X_{i}$ generate analytic semigroups of bounded linear operators $T_{N}(t)$ and $T_{P}(t)$ for $t \geq 0$ such that $N(t)=T_{N}(t) \varphi_{1}$ and $P(t)=T_{P}(t) \varphi_{2}$ are solutions of the abstract linear differential equations in $X_{i}$ given by

$$
N^{\prime}(t)=A_{N} N(t), P^{\prime}(t)=A_{P} P(t) .
$$

An additional property of the semigroup is that for each $t>0, T_{N}(t)$ and $T_{P}(t)$ are compact operators. In the language of partial differential equations $N(x, t)=$ $\left[T_{N}(t) \varphi_{1}\right](x)$ and $P(x, t)=\left[T_{P}(t) \varphi_{2}\right](x)$ are classical solutions of the initial boundary value problem (2.2) with $F_{1}=F_{2}=0$.

Let $\mathcal{T}(t): X \rightarrow X$ be defined by $\mathcal{T}(t)=T_{N}(t) \times T_{P}(t)$. Then $\mathcal{T}(t)$ is a semigroup of operators on $X$ generated by the operator $\mathcal{A}=A_{N} \times A_{P}$ defined on $D(\mathcal{A})=$ $D\left(A_{N}\right) \times D\left(A_{P}\right)$ and $U(x, t)=[\mathcal{T}(t) \varphi](x)$ is the solution of the linear system

$$
\begin{aligned}
& \frac{\partial U}{\partial t}(x, t)=D \Delta U(x, t), \quad x \in \Omega, \quad t>0 \\
& \frac{\partial U}{\partial \eta}(x, t)=0, \quad x \in \partial \Omega, \quad t>0, \quad U(x, 0)=\varphi(x), \quad x \in \Omega .
\end{aligned}
$$

Observe that the nonlinear term $F$ is twice continuously differentiable in $U$. Therefore, we can define the map $\left[F^{*}(\varphi)\right](x)=F(\varphi(x))$ which maps $X$ into itself and equation (2.2) can be viewed as the abstract ordinary differential equation in $X$ given by

$$
u^{\prime}(t)=\mathcal{A} u(t)+F^{*}(u(t)), u(0)=\varphi .
$$

While a solution $u(t)$ de (2.3) can be obtained under the restriction that $\varphi \in D(\mathcal{A})$, a mild solution can be obtained for every $\varphi \in X$ by requiring only that $u(t)$ is a 
continuous solution of the following integral equation

$$
u(t)=\mathcal{T}(t) \varphi+\int_{0}^{t} \mathcal{T}(t-s) F^{*}(u(s)) d s, \quad t \in[0, \beta),
$$

where $\beta=\beta(\varphi) \leq \infty$. Restricting our attention to functions $\varphi$ in the set

$$
X_{\Lambda}=\{\varphi \in X: \varphi(x) \in \Lambda, x \in \bar{\Omega}\},
$$

where $\Lambda=\left\{U=(N, P) \in R^{2}: N \geq 0, P \geq 0\right\}$, and taking into account the definition of the functions $F_{i}$, we obtain that $F_{1}(0, P)=0$ and $F_{2}(N, 0)=0$ for $U \in \Lambda$. Thus, Corollary 3.2 from [16, p. 129] implies that the Nagumo condition for the positive invariance of $\Lambda$ is satisfied, i. e.,

$$
\lim _{h \rightarrow 0^{+}} h^{-1} \operatorname{dist}(\Lambda, U+h F(U))=0, \quad U \in \Lambda .
$$

On the other hand, the direct application of the strong parabolic maximum principle can be used to show that the linear semigroup $\mathcal{T}(t)$ leaves $X_{\Lambda}$ positively invariant, i. e.,

$$
\mathcal{T}(t) X_{\Lambda} \subset X_{\Lambda}, \quad t \geq 0 .
$$

Finally, conditions (2.5) and (2.6) together allow us to apply Theorem 3.1 from [16, p. 127], which gives us

Lemma 1. For each $\varphi \in X_{\Lambda}$, (2.1) has a unique mild solution $u(t)=u(\varphi, t) \in$ $X_{\Lambda}$ and a classical solution $U(x, t)=[u(t)](x)$. Moreover, the set $X_{\Lambda}$ is positively invariant under the flow $\Psi_{t}(\varphi)=u(\varphi, t)$ induced by $(2.1)$.

So, the model (2.1) is biologically well-posed and its relevant dynamic is concentrated in $X_{\Lambda}$.

Finally, we are going to prove that all solutions of system (2.1) are bounded and therefore defined for all $t \geq 0$. Actually, from the following result by using the general theory of infinite dynamical system it follows that the relevant dynamic of the system (2.1) is concentrated in a compact set of the space $X_{\Lambda}$.

Theorem 1. Let $(N, P)$ be any solution of (2.1). Then

$$
\limsup _{t \rightarrow \infty} \max _{x \in \Omega} N(x, t) \leq 1, \quad \limsup _{t \rightarrow \infty} \max _{x \in \Omega} P(x, t) \leq \frac{1}{r} .
$$

Proof. From the first equation of the system (2.1), it follows that

$$
\frac{\partial N}{\partial t} \leq d_{1} \Delta N+N(1-N)
$$

as long $N$ is defined as a function of $t$.

Let $z$ be the solution of the equation

$$
z^{\prime}(t)=z(t)(1-z(t)), \quad z(0)=\max _{x \in \Omega} N(x, 0) .
$$


From the comparison principle, we obtain $N(x, t) \leq z(t)$. Now, taking into account that for any $\epsilon>0$ there exists a $T_{\epsilon}>0$ such that $z(t)<1+\epsilon$ for any $t \geq T_{\epsilon}$, which in turn implies that $N(x, t)$ is defined for all $t \geq 0$, and $\lim \sup _{t \rightarrow \infty} \max _{x \in \Omega} N(x, t) \leq 1$.

Having in mind that for a given $\epsilon>0$ there exists a $T_{\epsilon}>0$ such that $N(x, t) \leq 1+\epsilon$ for any $x \in \Omega$ and $t \geq T_{\epsilon}$, and by using the second equation of (2.1), we get

$$
\frac{\partial P}{\partial t}-d_{2} \Delta P \leq \delta P\left(-r+\frac{1+\epsilon}{P}\right)=-\delta r P+\delta(1+\epsilon)
$$

for any $x \in \Omega$ and $t \geq T_{\epsilon}$.

Let $z$ be the solution of the following initial value problem

$$
z^{\prime}(t)=-\delta r z(t)+\delta(1+\epsilon), \quad z\left(T_{\epsilon}\right)=\max _{x \in \Omega} P\left(x, T_{\epsilon}\right) .
$$

After a straightforward computation we get

$$
z(t) \leq \frac{1+\epsilon}{r}+z\left(T_{\epsilon}\right) e^{-\delta r\left(t-T_{\epsilon}\right)} \quad\left(\forall t \geq T_{\epsilon}\right) .
$$

Finally, by using the comparison principle we know that $P(x, t) \leq z(t)$ as long as $P$ is defined as a function of $t$. This, together with the previous inequality, implies that

$$
\limsup _{t \rightarrow \infty} \max _{x \in \Omega} P(x, t) \leq \frac{1}{r},
$$

which completes the proof.

\section{ANALYSIS OF THE MODEL WITHOUT DIFFUSION}

In this section we will study the system (2.1) without diffusion, i. e.,

$$
N^{\prime}(t)=F_{1}(N, P), \quad P^{\prime}(t)=F_{2}(N, P) .
$$

In particular, we will focus our attention on the existence of equilibria and their local stability. This information will be crucial in the next section where we study the effect of the diffusion parameters on the stability of the steady states.

The equilibria of the system (3.1) are given by the solution of the following equations

$$
N\left(1-N-\frac{s P}{P+N}\right)=0, \quad \delta P\left(-r+\frac{N}{P+N}\right)=0 .
$$

The system (3.1) has in the first quadrant the equilibrium points $(0,0)$ and $(1,0)$ for all values of the parameters. If $0<r<1$ and $0<s<1 /(1-r)$, then (3.1) admits a nontrivial equilibrium, which is given by

$$
\left(N^{*}, P^{*}\right)=\left(s(r-1)+1, \frac{(1-r)[s(r-1)+1]}{r}\right) .
$$

We point out that for $r=1$ we get that $\left(N^{*}, P^{*}\right)=(1,0)$. 
Hereafter, we will assume that $(r, s) \in D$, where $D$ is the region given by

$$
D=\left\{(r, s): 0<r<1,0<s<\frac{1}{1-r}\right\} .
$$

In the system (3.1), the origin is a non-analytical complicated equilibrium point. The structure of a neighborhood of point $(0,0)$ in the first quadrant of the plane $(x, y)$ and the asymptotes of trajectories for $x, y \rightarrow 0$ depend on parameter values and change in an essential way with a change of parameter (see [6]).

A straightforward computation shows us that the equilibrium point $(1,0)$ is locally asymptotically stable for $r>1$, and unstable if $0<r<1$.

Linearizing the system (3.1) around the nontrivial equilibrium $\left(N^{*}, P^{*}\right)$, we obtain that the characteristic equation is given by

$$
\lambda^{2}-\operatorname{trace} A \lambda+\operatorname{det} A=0
$$

where

$$
A=\left(\begin{array}{cc}
s\left(1-r^{2}\right)-1 & -s r^{2} \\
\delta(1-r)^{2} & -\delta r(1-r)
\end{array}\right) .
$$

Taking into account that $\operatorname{Re} \lambda<0$ if and only if trace $A<0$ and $\operatorname{det} A>0$, we get that $\left(N^{*}, P^{*}\right)$ is locally asymptotically stable if and only if $r, s, \delta \in D_{s}$, where $D_{s}$ is the set determined by the following inequalities:

$$
0<r<1, \quad 0<s<\frac{1}{1-r^{2}}+\frac{\delta r}{1+r}, \quad s<\frac{1}{1-r}, \quad \delta>0 .
$$

Let us set $f(r)=(1-r)^{-1}$ and

$$
g_{\delta}(r)=\frac{r \delta}{1+r}+\frac{1}{1-r^{2}}
$$

where $\delta$ is a positive parameter. We present on Fig. 1a and Fig. 1b the regions of the asymptotic stability of the nontrivial equilibrium.

\section{Turing InStability}

It is obvious that the equilibria of the system (3.1) are solutions of (2.1). We shall focus our attention on the nontrivial equilibrium $U^{*}=\left(N^{*}, P^{*}\right)$ of the system (3.1). More concretely, in this section we will analyze the stability of nontrivial steady-state solutions of (2.1).

Definition 1 (see [14]). The equilibrium $U^{*}$ of (2.1) is said to be diffusionally (Turing) unstable if it is an asymptotically stable equilibrium of (3.1) but it is unstable with respect to (2.1).

The stability of a homogeneous stationary solution $U^{*}$ of (3.1) will be studied via the linearized stability analysis (see, e. g., [10, pp. 68-70]). Setting $W=U-U^{*}$ and 


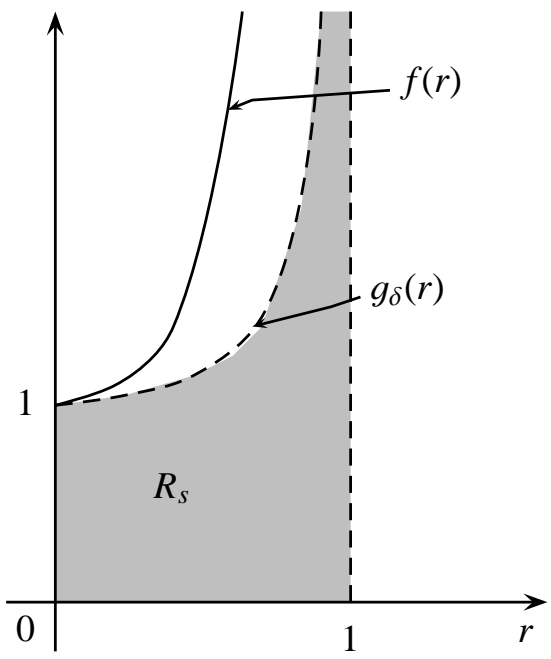

(a)

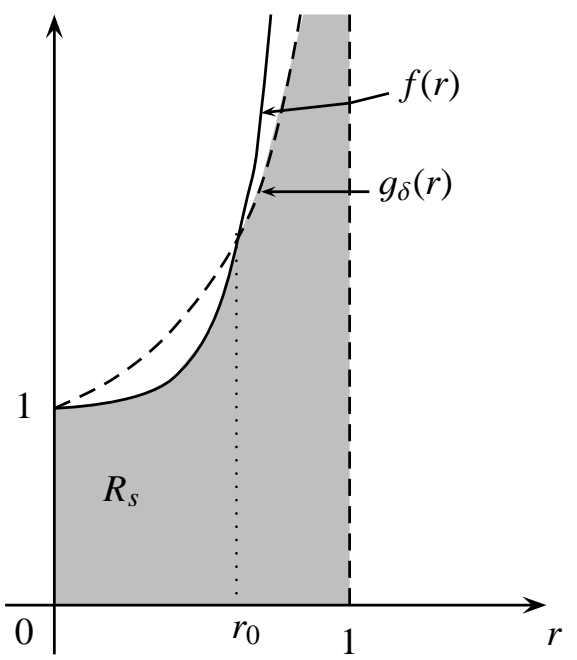

(b)

FiguRE 1. (a): $R_{S}$, the region of the local asymptotic stability for $0<\delta \leq 1$; (b): $R_{S}$, the region of the local asymptotic stability for $\delta>1$.

recalling that $A=F^{\prime}\left(U^{*}\right)$, as given previously, the linearized system of the reactiondiffusion equation (2.1) around $U^{*}$ is given by

$$
\frac{\partial W}{\partial t}=D \Delta W+A W, \quad \frac{\partial W}{\partial \eta}(x, t)=0, \quad x \in \partial \Omega, t>0 .
$$

The trivial solution, $W=0$, is asymptotically stable if and only if every solution of (4.1) decays to zero as $t \rightarrow \infty$.

Let $\phi_{j}(x)$ denote the $j$ th eigenfunction of the Laplacian operator $-\Delta$ on $\Omega$ with no-flux boundary conditions. That is,

$$
\Delta \phi_{j}+\lambda_{j} \phi_{j}=0, \quad x \in \Omega, \quad \boldsymbol{n} \cdot \nabla \phi_{j}=0, x \in \partial \Omega,
$$

for scalars $\lambda_{j}$ satisfying

$$
0=\lambda_{0}<\lambda_{1}<\lambda_{2}<\ldots
$$

The determination of the pairs $\left(\phi_{j}, \lambda j\right)$ is a standard problem (see, e. g., [9, pp. 205208]). The differential operator $-\Delta$, with no-flux boundary conditions, is self-adjoint in $L_{2}(\Omega)$, i. e.,

and it is easy to see that

$$
\int_{\Omega}-\Delta \psi_{1} \cdot \psi_{2} d x=\int_{\Omega}-\Delta \psi_{2} \cdot \psi_{1} d x
$$

$$
\lambda_{j}=\frac{\int_{\Omega}\left|\nabla \phi_{j}\right|^{2} d x}{\int_{\Omega} \phi_{j}^{2} d x}>0
$$


for all $j \geq 1$. We can suppose without loss of generality that the $\phi_{j^{\prime} s}$ are normalized so that $\left\|\phi_{j}\right\|_{L_{2}(\Omega)}=1$. Moreover, the set of $\phi_{j}$ forms an orthogonal basis for $L_{2}(\Omega)$ and any function may be expanded as a Fourier series or eigenfunction expansion

$$
u(x)=\sum_{j=0}^{\infty} u_{j} \phi_{j}(x) .
$$

Using these preliminaries, we can solve (4.1) by expanding our solution $W$ via

$$
W(x, t)=\sum_{j=0}^{\infty} s_{j}(t) \phi_{j}(x)
$$

where each $s_{j}(t) \in \mathbb{R}^{2}$. Substituting (4.2) into (4.1) and equating the coefficients at every $\phi_{j}$, we have

where $B_{j}$ is the matrix

$$
\frac{d s_{j}}{d t}=B_{j} s_{j}
$$

$$
B_{j}=A-\lambda_{j} D
$$

Now the trivial solution $W=0$ of (4.1) is asymptotically stable if and only if each $s_{j}(t)$ decays to zero as $t \rightarrow \infty$. This is equivalent to the condition that each $B_{j}$ has two eigenvalues with negative real parts for all $j$. The eigenvalues of the matrix $B_{j}$ are given by

$$
\operatorname{det}\left[B_{j}-\varrho I\right]=\varrho^{2}-\operatorname{trace} B_{j} \varrho+\operatorname{det} B_{j}=0 .
$$

Hereafter, we are going to assume that parameters $r, s, \delta \in D_{s}$; i. e. $r, s, \delta$ belong to the region where the nontrivial equilibrium $U^{*}$ of the system (3.1) is asymptotically stable. Now we shall study the stability of $U^{*}$ with respect to the system (2.1) in the $\left(d_{1}, d_{2}\right)$ plane.

Taking into account that $r, s, \delta \in D_{s}$, it follows that trace $A<0$ and $\operatorname{det} A>0$. Therefore, $\operatorname{trace} B_{j}=\operatorname{trace} A-\lambda_{j}\left(d_{1}+d_{2}\right)<0$, due to $\lambda_{j} \geq 0, j=0,1,2, \ldots$, and $d_{1}, d_{2}>0$. Henceforth, for the Turing instability to occur, it should be satisfied that $\operatorname{det} B_{j} \leq 0$, for some $j \geq 1$, where $\operatorname{det} B_{j}=\left(A_{11}-\lambda_{j} d_{1}\right)\left(A_{22}-\lambda_{j} d_{2}\right)-A_{12} A_{21}$.

For fixed $\lambda$ let us denote the hyperbola in the $\left(d_{1}, d_{2}\right)$ plane by

$$
H_{\lambda}:\left(\lambda d_{1}-A_{11}\right)\left(\lambda d_{2}-A_{22}\right)-A_{12} A_{21}=0 .
$$

We know that $A_{22}=-\delta r(1-r)<0$ on the admissible region. Hence, the location of the graph of the hyperbola $H_{\lambda}$ on the $\left(d_{1}, d_{2}\right)$-plane is dictated by the sign of $A_{11}=$ $s\left(1-r^{2}\right)-1$. A straightforward computation gives us that the graph of the function $h(r)=1 /\left(1-r^{2}\right)$ lies strictly below the boundary of the region of asymptotic stability for any $\delta>0$, see Fig. 2a and Fig. $2 b$.

Let us suppose that $A_{11}<0$. In this case $\operatorname{det} B_{j}>0$ for any $j \geq 0$ and $d_{1}>0$, $d_{2}>0$. We disregard this situation because we are looking for conditions of the Turing instability, see Fig. 3a. Assuming that $A_{11}>0$, we obtain that there exist 


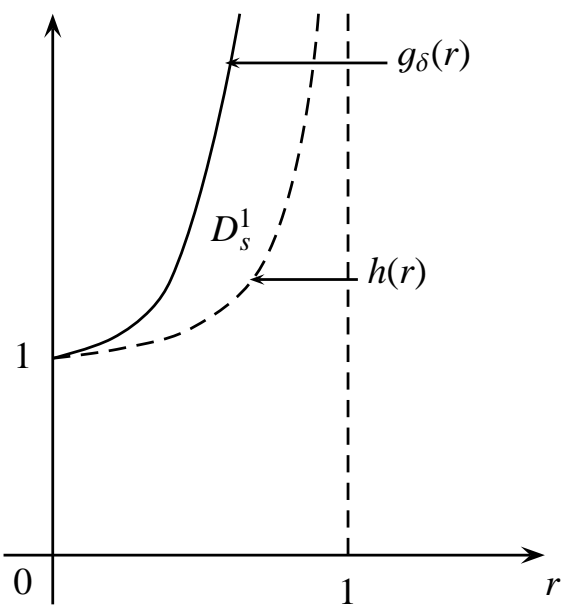

(a)

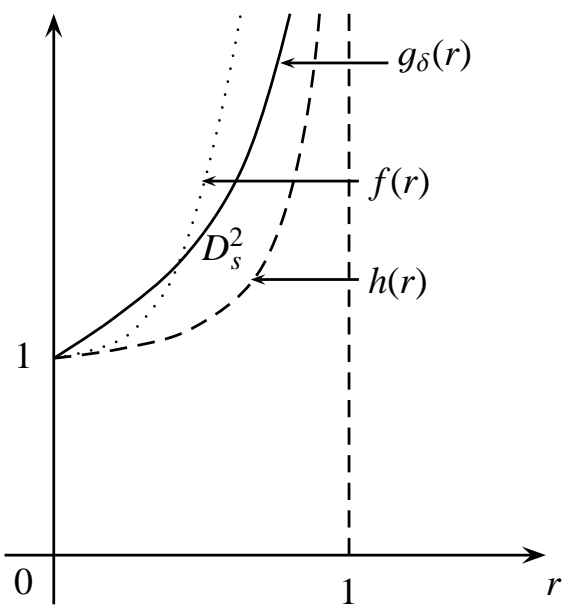

(b)

Figure 2. (a): $0<\delta \leq 1$; (b): $\delta>1$.

positive parameters $d_{1}$ and $d_{2}$ where $U^{*}$ is diffusionally unstable. That region is depicted on Fig. $3 b$.

From Fig. 3b, it follows that the set of $\left(d_{1}, d_{2}\right) \in \mathbb{R}_{+}^{2}$ satisfying that det $B_{j} \leq 0$ for some $j \in \mathbb{N}$ consists of all points which are above the graph of the hyperbola $H_{\lambda_{j}}$. Clearly, for each $j \in \mathbb{N}$ this set is nonempty and therefore we can always choose $\left(d_{1}, d_{2}\right) \in \mathbb{R}_{+}^{2}$ in such a way that $U^{*}$ is diffusionally unstable. Let us fix $d_{2}>0$. Since $\lambda_{j} \rightarrow \infty$ as $j \rightarrow \infty$, then there exists a $k \in \mathbb{N}$ such that $d_{k}^{*}=\frac{\operatorname{det} A}{\lambda_{k} A_{11}}<d_{2}$. Therefore, the point $\left(d_{A}, d_{2}\right)$ belongs to the hyperbola $H_{\lambda_{k}}$, where

$$
d_{A}=\frac{A_{11} \lambda_{k} d_{2}-\operatorname{det} A}{\lambda_{k}\left(\lambda_{k} d_{2}-A_{22}\right)} .
$$

Moreover, if $0<d_{1}<d_{A}$, then $\left(d_{1}, d_{2}\right)$ will lie above the graph of $H_{\lambda_{k}}$ and the homogeneous steady-state solution $U^{*}=\left(N^{*}, P^{*}\right)$ will be diffusionally unstable. We can also remark that if $d_{2} \rightarrow \infty$, we have

$$
\frac{A_{11} \lambda_{k} d_{2}-\operatorname{det} A}{\lambda_{k}\left(\lambda_{k} d_{2}-A_{22}\right)} \rightarrow \frac{A_{11}}{\lambda_{k}}
$$

\section{Pattern formation}

In this section we shall show how the diffusion-driven instability phenomenon gives rise to nonhomogeneous steady-state solutions of (2.1) that bifurcate from the uniform stationary solution. For this purpose, we start by introducing a definition. 


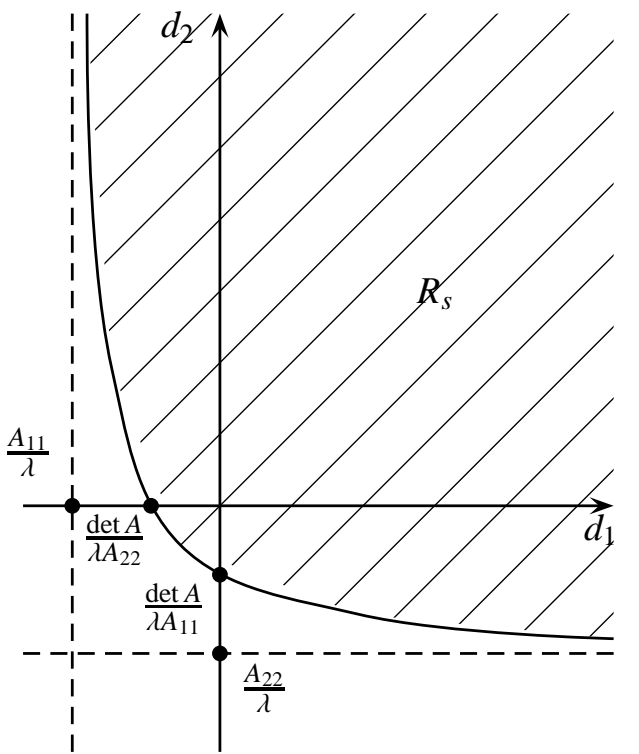

(a)

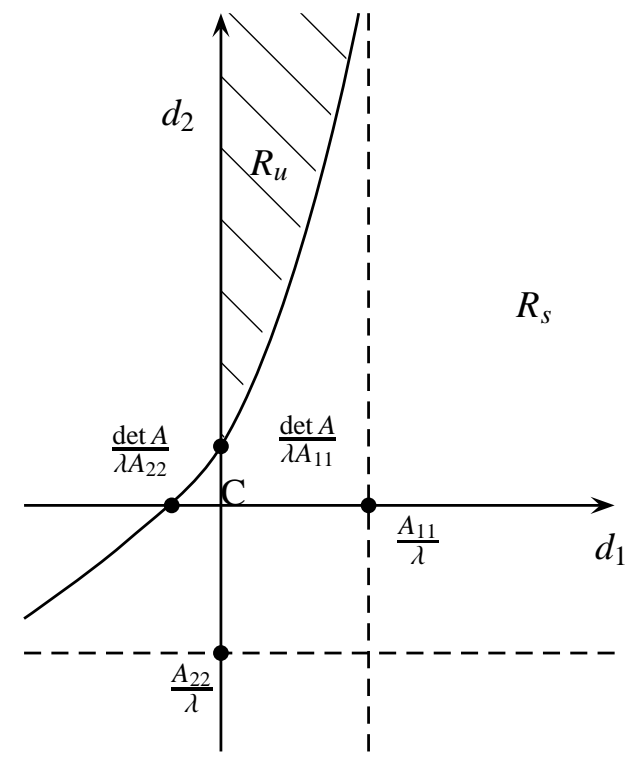

(b)

Figure 3. (a): $R_{s}$, the stability region for $A_{11}<0, r, s, \delta \in D_{s}$, and $\lambda>0$; (b): $R_{s}$, the stability region, and $R_{u}$, the instability region for $A_{11}>0, r, s, \delta \in D_{s}$, and $\lambda>0$.

Consider the following reaction-diffusion system

$$
\frac{\partial U}{\partial t}=D \Delta U+F(U), \quad \frac{\partial U}{\partial \eta}(x, t)=0, \quad x \in \partial \Omega, t>0
$$

where $U \in \mathbb{R}^{2}, D$ is a $2 \times 2$ nonnegative diagonal matrix and $F: \mathbb{R}^{2} \rightarrow \mathbb{R}^{2}$ is a smooth function, where $\partial / \partial \eta$ denotes the normal derivate. Assume that $U^{*}$ is an uniform stationary solution of (5.1), i. e., $F\left(U^{*}\right)=0$.

Definition 2. We say that $U^{*}$ undergoes a Turing bifurcation at $\mu_{0} \in(0, \infty)$ if the solution $U^{*}$ changes its stability at $\mu_{0}$ and in some neighborhood of $\mu_{0}$ there exists a one-parameter family of nonconstant stationary solution of systems (5.1).

Now we use Theorem 13.5 from [17] for to determine the nonhomogeneous stationary solutions of (5.1), in this case take $d_{2}$ as bifurcation parameter.

Theorem 2. Let $v_{1 k}$ and $v_{2 k}$ be the eigenvectors of $B_{k}$ corresponding to the eigenvalues $\lambda_{1 k}$ and $\lambda_{2 k}$, respectively. Assume that

(1) $r, s, \delta \in D_{s}^{i}, i=1,2$,

(2) $v_{1 k}=\left(\begin{array}{l}\xi_{1} \\ \xi_{2}\end{array}\right)$ and $v_{2 k}$ is not parallel to $\left(\begin{array}{c}\xi_{1} \\ 0\end{array}\right)$,

(3) $0<d_{1}<D^{*}$, where $D^{*}=A_{11} \lambda_{k}^{-1}$. 
Then there exists a $k \in \mathbb{N}$ such that at

$$
d_{2}^{*}=\frac{A_{22} \lambda_{k} d_{1}-\operatorname{det} A}{\lambda_{k}\left(\lambda_{k} d_{1}-A_{11}\right)}
$$

the uniform steady-state solution $U^{*}$ of (5.1) undergoes the Turing bifurcation.

Proof. Hereafter, the role of the space $X$ will be played by

$$
X=\left\{W \in C\left(\Omega, \mathbb{R}^{2}\right) \times C\left(\Omega, \mathbb{R}^{2}\right): \frac{\partial W}{\partial \eta}(x, t)=0, t>0 x \in \partial \Omega\right\}
$$

with the supremum norm involving the first and second derivatives, and $Y=C\left(\Omega, \mathbb{R}^{2}\right)$ with the usual supremum norm. However, when choosing the subspace $Z$, we shall use the orthogonality induced by the scalar product

$$
\langle V, W\rangle=\int_{\Omega}\left(V_{1}(x) W_{1}(x)+V_{2}(x) W_{2}(x)\right) d x,
$$

where $V=\left(V_{1}, V_{2}\right)$ y $W=\left(W_{1}, W_{2}\right)$.

Setting $W=U-U^{*}$, where $U^{*}$ is a nontrivial homogeneous steady-state solution of (5.1), we get

$$
W_{t}=D \Delta W+A W+G(W), \quad \frac{\partial W}{\partial \eta}(x, t)=0, \quad t>0, \quad x \in \partial \Omega,
$$

where $A$ is the Jacobian matrix of $F$ in $U^{*}$ and $G(W)=F\left(U^{*}+W\right)-A W$.

For any nonhomogeneous stationary solution $U$ of (5.1), $W=U-U^{*}$ satisfies the elliptic equation

$$
D \Delta W+A W+G(W)=0, \quad \frac{\partial W}{\partial \eta}(x, t)=0, t>0 x \in \partial \Omega .
$$

Taking into account this observation, define the function $f: \mathbb{R} \times X \rightarrow Y$ and linear operator $L_{0}$ considered in Theorem 13.5 of [17] as follows: $f\left(d_{2}, W\right)=D \Delta W+A W+$ $G(W)$ and

$$
L_{0}=D_{2} f\left(d_{2}^{*}, 0\right)=\frac{\partial f\left(d_{2}^{*}, 0\right)}{\partial W},
$$

where $d_{2}$ is the diffusion coefficient of the susceptible class. The spectrum of the linear operator $L_{0}$ is given by the eigenvalues $\lambda_{i j}$ of the matrices

$$
B_{j}=A-\lambda_{j} D
$$

evaluated at $d_{2}=d_{2}^{*}$, where $i=1,2$, and $j=0,1,2, \ldots$. Since $0<d_{1}<D^{*}$, there exists a unique $k \in \mathbb{N}$ such that $\left(d_{1}, d_{2}^{*}\right)$ belongs to the hyperbola $H_{\lambda_{k}}$. 


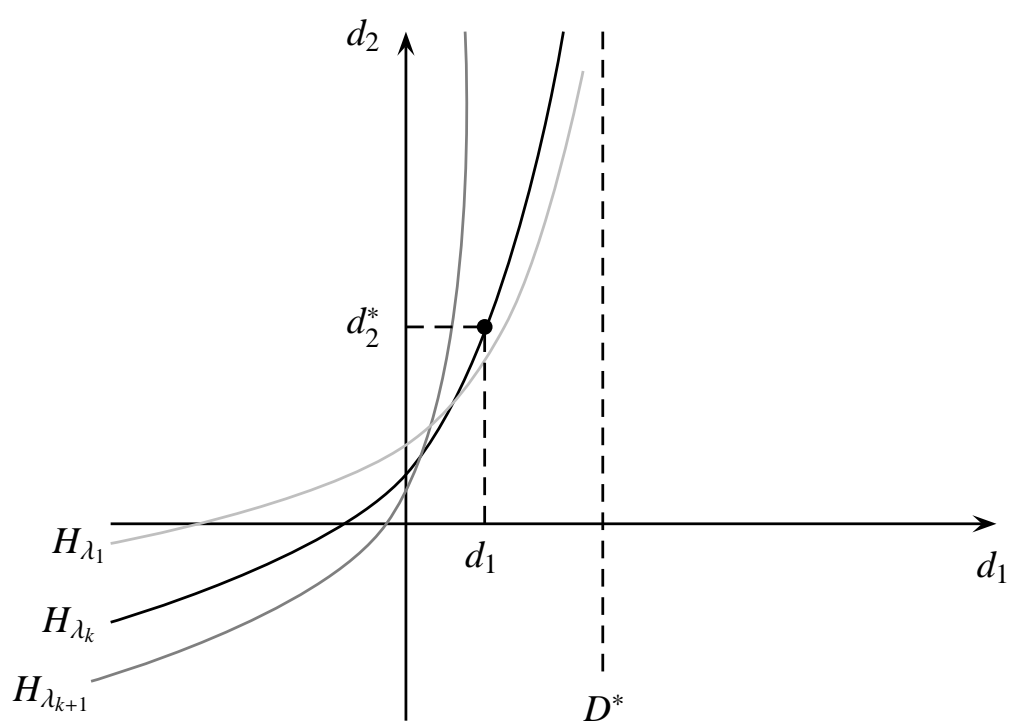

Figure 4. Turing Bifurcation. When $d_{1}<D^{*}$, the uniform steadystate solution $U^{*}$ of (5.1) undergoes a Turing bifurcation at $d_{2}=d_{2}^{*}$

In other words, $\operatorname{det} B_{j}>0$ for $j \neq k$ and det $B_{j}=0$ just for $j=k$. Therefore, for $i=1,2$ and $j=0,1,2, \ldots, k-1, k+1, \ldots$ all eigenvalues $\lambda_{i j}$ have negative real parts. For $j=k$, one eigenvalue, say $\lambda_{1 k}$, is zero and the other one is negative, i. e., $\lambda_{2 k}<0$.

Since $v_{1 k}$ is the eigenvector of $B_{k}$ corresponding to the zero eigenvalue $\lambda_{1 k}$, the eigenfunction of the linear operator $L_{0}$ corresponding to $\lambda_{1 k}=0$ is given by $\psi_{k}=$ $v_{1 k} \Phi_{k}(x)$ which is a non-uniform stationary solution of the linearized system (4.1), i. e.,

$$
D \Delta \psi_{k}(x)+A \psi_{k}(x)=0, \quad \frac{\partial \psi_{k}}{\partial \eta}(x)=0, x \in \partial \Omega
$$

Therefore, the null subspace $N\left(L_{0}\right)$ of the operator $D_{2} f\left(d_{2}^{*}, 0\right)$ is one-dimensional, spanned by $\psi_{k}$. Because of the orthogonality of the system, $\Phi_{n}(x), n=0,1,2, \ldots$ obtained by solving the eigenvalue problem

$$
\begin{gathered}
\Delta \Phi_{n}(x)+\lambda_{n} \Phi_{n}(x)=0, \quad x \in \Omega, \\
\boldsymbol{n} . \nabla \Phi_{n}(x)=0, \quad x \in \partial \Omega .
\end{gathered}
$$

The range $R\left(L_{0}\right)$ of this operator is given by the relation

$$
R\left(L_{0}\right)=\left\{U \in[C(\Omega, \mathbb{R})]^{2}: \text { the Fourier expansion of } U\right.
$$

does not contain the term $\left.\Phi_{n}(x)\right\} \cup\left\{v_{2 k} \Phi_{n}(x)\right\}$, 
and has codimension one. So conditions (i) and (ii) of Theorem 13.5 from [17] are satisfied. It still remains to verify condition (iii). Let

$$
L_{1}=D_{1} D_{2} f\left(d_{2}^{*}, 0\right)=\frac{\partial}{\partial d}\left(\frac{\partial f}{\partial W}\right)\left(d_{2}^{*}, 0\right) .
$$

Then $L_{1}=\left(\begin{array}{cc}\Delta & 0 \\ 0 & 0\end{array}\right)$ and

$$
L_{1} \psi_{k}=\left(\begin{array}{cc}
\Delta & 0 \\
0 & 0
\end{array}\right) v_{1 k} \Phi_{n}(x)=-\lambda_{n}\left(\begin{array}{c}
\xi_{1} \\
0
\end{array}\right) \Phi_{n}(x),
$$

with $\xi_{1} \neq 0$, and $\left(\begin{array}{c}\xi_{1} \\ 0\end{array}\right)$ not being parallel to $v_{2 k}$. Then, $L_{1} \psi_{1 k} \notin R\left(L_{0}\right)$ and condition (iii) of Theorem 13.5 from [17] is satisfied. So, by choosing $Z=R\left(L_{0}\right)$ we conclude that there exists a $\gamma>0$ and a $C^{1}$ curve $(d, \phi):(-\gamma, \gamma) \rightarrow \mathbb{R} \times Z$ with $d(0)=d_{2}^{*}$ and $\phi(0)=0$ such that

$$
W(x, s)=s v_{1 k} \Phi_{n}(x)+s \phi(x, s)
$$

is a solution of the elliptic equation (5.3) with $d_{2}=d(s), s \in(-\gamma, \gamma)$. Finally, taking into account that $W=U-U^{*}$, we obtain

$$
U(x, s)=U^{*}+s v_{1 k} \Phi_{n}(x)+O\left(s^{2}\right)
$$

are non-uniform stationary solutions of (5.1) with $d_{2}=d(s)$, and $s \in(-\gamma, \gamma)$.

Therefore, at $d_{2}=d_{2}^{*}$, the uniform steady-state solution $U^{*}$ undergoes a Turing bifurcation.

\section{STABility Of BifURCATing SOlution}

In this section we will study the stability of the one parameter family of nonuniform stationary solution $U(x, s)$ of the system (2.1) that arise from the bifurcation of the homogeneous steady state $U^{*}$.

We showed that $\lambda_{1 k}$ is a $L_{1}$-simple eigenvalue of $L_{0}$, where $L_{1}=D_{1} D_{2} f\left(d_{2}^{*}, 0\right)$ and $L_{0}=D_{2} f\left(d_{2}^{*}, 0\right)$. On the other hand, for $|\varepsilon|$ and $|s|$ small enough, the operators $D_{2} f\left(d_{2}^{*}+\varepsilon, 0\right)$ and $D_{2} f\left(d(s), s \psi_{k}+s \phi(x, s)\right)$ are close to $L_{0}$. Applying Lemma 13.7 of [17], we obtain that there exist functions

$$
d \longmapsto\left(\varrho(d), \psi_{c}(d)\right), \quad s \longmapsto\left(\eta(s), \psi_{b}(s)\right)
$$

defined on neighborhoods of $d_{2}^{*}$ and 0 , respectively, such that

$$
\begin{gathered}
D_{2} f(d, 0) \psi_{c}(d)=\varrho(d) \psi_{c}(d), \\
D_{2} f\left(d(s), s \psi_{k}+s \phi(x, s)\right) \psi_{b}(s)=\eta(s) \psi_{b}(s),
\end{gathered}
$$

and

$$
\left(\varrho\left(d_{2}^{*}\right), \psi_{c}\left(d_{2}^{*}\right)\right)=\left(0, \psi_{k}\right)=\left(\eta(0), \psi_{b}(0)\right) .
$$


Note that the functions

$$
\begin{aligned}
\eta(s) & =\eta\left(D_{2} f\left(d(s), s \psi_{k}+s \phi(x, s)\right)\right), \\
\psi_{b}(s) & =\psi_{b}\left(D_{2} f\left(d(s), s \psi_{k}+s \phi(x, s)\right)\right), \\
\varrho(d) & =\eta\left(D_{2} f(d, 0)\right), \quad \psi_{c}(d)=\psi_{b}\left(D_{2} f(d, 0)\right)
\end{aligned}
$$

given by Lemma 13.7 of [17] are smooth functions.

The following result is the Crandall-Rabinowitz Theorem 1.16 from [8, p. 165].

Theorem 3. Let the assumptions of Theorem 13.5 of [17] hold, and let the functions $\varrho(d)$ and $\eta(s)$ be defined as above. Then $\varrho^{\prime}\left(d_{2}^{*}\right) \neq 0$, and if $\eta(s) \neq 0$ for s close to 0 , then

$$
\lim _{s \rightarrow 0} \frac{s d^{\prime}(s) \varrho^{\prime}\left(d_{2}^{*}\right)}{\eta(s)}=-1 .
$$

First we determine $\varrho^{\prime}\left(d_{2}^{*}\right)$. It is known that $\varrho\left(d_{2}\right)$ satisfies the equation

$$
\varrho^{2}\left(d_{2}\right)-\operatorname{trace} B_{k} \varrho\left(d_{2}\right)+\operatorname{det} B_{k}=0 .
$$

Differentiating implicitly the former equation with respect to $d_{2}$, we have

$$
\varrho^{\prime}\left(d_{2}\right)=\frac{\lambda_{k} A_{11}-\lambda_{k}^{2} d_{1}-\lambda_{k} \varrho\left(d_{2}\right)}{2 \varrho\left(d_{2}\right)-\operatorname{trace} B_{k}} .
$$

Evaluating at $d_{2}^{*}$, we obtain

$$
\varrho^{\prime}\left(d_{2}^{*}\right)=\frac{\lambda_{k}^{2} d_{1}-\lambda_{k} A_{11}}{\operatorname{trace} A-\lambda_{k}\left(d_{1}+d_{2}^{*}\right)}=\frac{\lambda_{k}\left(\lambda_{k} d_{1}-A_{11}\right)}{\operatorname{trace} A-\lambda\left(d_{1}+d_{2}^{*}\right)} .
$$

Since $A_{11}>0$ and $0<d_{1}<A_{11} \lambda_{k}{ }^{-1}$, we see that $\lambda_{k} d_{1}-A_{11}<0$ and trace $A-\lambda_{k}\left(d_{1}+\right.$ $\left.d_{2}^{*}\right)<0$. Therefore,

$$
\varrho^{\prime}\left(d_{2}^{*}\right)>0 .
$$

Proposition. Let $(d(s), U(x, s))$ be the one parameter family of bifurcating solutions given by the formula

$$
U(x, s)=W^{*}+s v_{1 k} \Phi_{n}(x)+O\left(s^{2}\right) .
$$

Assume that the conditions of Theorem 2 are satisfied, $d^{\prime}(0) \neq 0$, and that the eigenvalues $\eta(s)$ of the nonhomogeneous steady state bifurcating from the critical value $\lambda_{1 k}=0$ are non-zero for small $|s| \neq 0$. Then if $d(s)<d_{2}^{*}$ the corresponding solution $U(x, s)$ is stable and if $d(s)>d_{2}^{*}$, the corresponding solution $U(x, s)$ is unstable.

Proof. We know that $\varrho^{\prime}\left(d_{2}^{*}\right)>0$. Let us determine the sign of $\eta(s)$. Since $d^{\prime}(0) \neq$ 0 , we may assume that $d^{\prime}(0)>0$. Then by continuity we have that $d^{\prime}(s)>0$ for $|s|$ small enough. Therefore, using (6.1), it follows that $\eta(s)<0$ for $s>0$ small enough, which in turn implies that the bifurcating solution is asymptotically stable. For small $s<0, \eta(s)>0$. Hence, the bifurcating nonhomogeneous stationary solution is unstable. 
The case $d(s)<0$ can be analyzed similarly. This completes the proof of our claim.

\section{Discussion}

In this paper, we discussed the main mathematical features exhibited by the reactiondiffusion system (1.1). More concretely, we showed that when $A_{11}=s\left(1-r^{2}\right)-1$ is positive, a nontrivial geotemporal dynamics of the reaction-diffusion ratio-dependent predator-prey model (1.1) can be obtained. In the case where the $0<d_{1}<A_{11} / \lambda_{j}$, we showed that for a wide range of parameter values and diffusion coefficients $d_{1}$ and $d_{2}$, see Fig. 3b, the nonlinear system (1.1) can exhibit stable spatially heterogeneous solutions which arise from Turing bifurcations. It is worth pointing out that a Turing bifurcation can not occur for a large diffusive coefficient of the prey, nevertheless the diffusive coefficient of the predator can be large enough.

The existence of this pattern formation for system (1.1) shows that the reactiondiffusion ratio-dependent predator-prey model exhibits features which were not possible for the classical model. More specifically, one can show that for a classical Lotka-Volterra prey-predator system with diffusion on a finite domain and zero flux, boundary condition cannot give rise to temporally or spatially inhomogeneous solutions asymptotically as $t \rightarrow \infty$.

In conclusion, we note that the mathematical analysis of model (1.1) shows how a reaction-diffusion ratio-dependent predator-prey model can stably regulate its growth around either spatially homogeneous or heterogeneous solutions through a Turing instability mechanism.

\section{ACKNowledgement}

We are deeply indebted to Professor Miklós Farkas for his detailed criticism that helped us to improve this paper.

\section{REFERENCES}

[1] Aкcakaya, H.R., Arditi, R., AND Ginzburg, L.R.: Ratio-dependent prediction: an abstraction that works, Ecology, 76 (1995), 995-1004.

[2] Arditi, R. and Berryman, A. A.: The biological paradox, Trends in Ecology and Evolution, 6 (1991), 32.

[3] Arditi, R. and Ginzburg, L. R.: Coupling in predator-prey dynamics: ratio-dependence, J. Theor. Biol., 139 (1989), 311-326.

[4] Arditi, R., Ginzburg, L. R., and Aкcakaya, H. R.: Variation in plankton densities among lakes: a case for ratio-dependent models, American Naturalist, 138 (1991), 1287-1296.

[5] Bartumeus, F., Alonso, D., and Catalan, J.: Self-organized spatial structures in a ratio dependent predator-prey model, Physica, A295 (2001), 53-57.

[6] Berezovskaya, F., Karev, G., and Arditi, R.: Parametric analysis of the ratio-dependent predatorprey model, J. Math. Biol., 43 (2001), 221-246.

[7] Cosner, C., DeAngelis, D. L., Ault, J. S., And Olson, D. B.: Effects of Spatial Grouping on the Functional Response of Predators, Theor. Pop. Biol., 56 (1999), 65-75. 
[8] Crandall, M. G. and Rabinowitz, P. H.: Bifurcation, perturbation of simple eigenvalues, and linearized stability, Arch. Rat. Mech. Anal., 52 (1973), 161-181.

[9] Folland, G. B.: Introduction to Partial Differential Equations, Princenton Univ. Press, 1976.

[10] Grindrod, P.: Patterns and Waves. The theory and Applications of Reaction-Diffusion Equations, Clarendon Press, Oxford, 1991.

[11] Hsu, S. B., Hwang, T. W., and Kunn, Y.: Global analysis of the Michaelis-Menten-type ratiodependent predator-prey system, J. Math. Biol., 42 (2001), 489-506.

[12] Jost, C., Arino, O., AND ARditi, R.: About deterministic extinction in ratio-dependent predatorprey models, Bull. of Math. Biol., 61 (1999), 19-32.

[13] KuAng, Y. and Beretta, E.: Global qualitative analysis of a ratio-dependent predator-prey system, J. Math. Biol., 36 (1998), 389-406.

[14] Окиво, А.: Diffusion and Ecological Problems: Mathematical Models, Springer, New York, etc., 1980.

[15] PANG, Y. H. P. AND WANG, M.: Qualitative analysis of a ratio-dependent predator-prey system with diffusion, Proc. Roy. Soc. Edinbourg, 133A (2003), 919-942.

[16] Sмiтн H. L.: Monotone Dynamical Systems. An Introduction to the Theory of Competitive and Cooperative Systems, Amer. Math. Soc., Providence, RI, 1995.

[17] Smoller J.: Shock Waves and Reaction-Diffusion Equations, Springer, New York, etc., 1983.

\section{Authors' addresses}

\section{Marcos Lizana:}

Departamento de Matemáticas, Facultad de Ciencias, Universidad de los Andes, La Hechicera,

Mérida, Edo. Mérida, Venezuela

E-mail address: lizana@ula.ve

\section{Julio J. Marín V.:}

Departamento de Matemática, Universidad de Oriente, Av. Universidad, Cumaná, Edo. Sucre, Venezuela

E-mail address: jjmarinv@hotmail.com 